\title{
WISSENSPOPULARISIERUNG UND -TRANSFER IN POPULÄRWISSENSCHAFTLICHEN TEXTEN. EINE TEXTLINGUISTISCHE ANALYSE DEUTSCHSPRACHIGER TEXTE IM BEREICH DER BIOLOGIE - EINE SKIZZE DES FORSCHUNGSVORHABENS
}

\begin{abstract}
1. Einleitung
Im folgenden Beitrag soll das Dissertationsvorhaben ${ }^{1}$ des Autors für den Zeitraum 2010-2014 vorgestellt werden. Der Titel der Dissertation deckt sich im Wortlaut mit dem Titel des vorliegenden Beitrags. Die Arbeit soll eine Analyse populärwissenschaftlicher Texte mit Heranziehung (fach)textlinguistischen Instrumentariums darstellen. Die Entscheidung für ein naturwissenschaftliches - und genauer biologisches - Textkorpus resultiert zum einen aus der großen Popularität solcher Publikationen (in traditioneller, gedruckter Form wie auch im Internet) und zum anderen subjektiv aus meinem eigenen Interesse an dieser Thematik. Noch als Schulkind wurde ich mit einer Reihe populärwissenschaftlicher Publikationen zur biologischen und ökologischen Thematik konfrontiert. Schon damals musste ich als Leser feststellen, dass sich manche von ihnen „leicht" lesen ließen, während andere den Eindruck verliehen, als wären sie allzu wissenschaftlich oder allzu informationsbeladen, kurz: kaum verständlich oder sogar unverständlich für einen Laien. Diese Tatsache kann als ein wichtiger Hinweis auf die Vertikalität der Texte/Diskurse gewertet werden.
\end{abstract}

\footnotetext{
${ }^{1}$ Ich hatte die Gelegenheit, es mit anerkannten Textlinguisten während der Konferenz in Słubice im Jahre 2011 zu besprechen. Für ihre kritischen Hinweise und Anregungen bin ich Herrn Prof. Wolfgang Heinemann und Herrn Prof. Stojan Bračič sehr dankbar. Die Dissertation ensteht unter Betreuung von Frau Prof. Beata Mikołajczyk am Lehrstuhl für Deutsche Sprache (Adam-Mickiewicz-Universität Poznań).
} 
Es lassen sich mehrere Faktoren nennen, die einen positiven oder negativen Einfluss auf die Verständlichkeit von populärwissenschaftlichen Publikationen haben, es sind u.a. Textlänge und -struktur, Terminologie oder Visualisierungen. Auch die Tatsache, dass viele Publikationen aus anderen Sprachen übersetzt werden, spielt hier eine Rolle. Unbestreitbar ist in dem Zusammenhang, dass man den Übersetzer mit zu der Gruppe der Popularisatoren des Wissens/der Wissenschaft zählen muss.

Die oben auch aus persönlicher Leser-Perspekve skizzierten Problemfelder sollen in der Dissertation ausführlich behandelt werden. Einen Überblick über die Hauptkapitel der Arbeit bieten die folgenden Abschnitte des Beitrags.

\section{Theoretischer Teil}

Im theoretischen Teil werden zuerst Überlegungen über Popularisierung angestellt. An dieser Stelle müssen kurz Geschichte, Definition, Zweck und Rolle der Popularisierung, Medien, über die das Wissen transferiert wird, u.v.a.m. angesprochen werden. Erwähnt werden müssen auch die Autoren populärwissenschaftlicher Texte - nicht selten sind es keine Wissenschaftler, sondern Wissenschaftsjournalisten. Es wird auch eine schwierige Frage gestellt danach, ob Popularisierung möglicherweise „gefährlich“ sein kann. Es wird mit anderen Worten gefragt, ob jeder Popularisierungsversuch geglückt sein muss ${ }^{2}$. Danach folgen Informationen zu kommunikativen Aspekten der Popularisierung. $\mathrm{Zu}$ verstehen sind darunter die verschiedenen Kommunikationskonstellationen, die zwischen Kommunikationspartnern (zwischen Textemittent und -rezipient) zustande kommen. Denken wir hier etwa an die Unterscheidung fachintern - interfachlich - fachextern von Möhn, Pelka (1984: 26) oder auch an die Aufteilung von Bongo (2006: 176), wo zum einen die Wissenschaftskommunikation in engerem Sinne und zum anderen die Populärwissenschaft genannt werden. In meinem Blickfeld befindet sich speziell die fachexterne Experten-Laien-Kommunikation ${ }^{3}$. Den Ausgangspunkt der Betrachtung kommunikativer Aspekte soll die Transferwissenschaft bilden, verstanden als

eine transdisziplinär orientierte Wissenschaft [...], die Prinzipien, Wege und Strategien des selektiven und nachhaltigen Zugangs zu Wissen im Zeitalter der Informationsflut und der Informationsexplosion erforschen soll. (Antos 2001: 5)

\footnotetext{
${ }^{2}$ Würde zudem die folgende Feststellung zutreffen: „Komplexe Inhalte bedingen oft einen komplizierten sprachlichen Ausdruck“ (Punkki-Roscher 1995: 35), so müsste man dann in Kauf nehmen, dass die Popularisierung nicht immer mit der Steigerung der Verständlichkeit gleichbedeutend sein kann.

${ }^{3}$ Die Dichotomie Experte - Laie ist zu allgemein, stattdessen sollte man von „Skalen von Experten- und Laienniveaus“" (Wichter 1999: 81) sprechen, dies würde einer vertikalen Schichtung entsprechen und die strenge Abgrenzung Experte vs. Laie meiden.
} 
Der vertikale Wissenstransfer (also vereinfachend: der Transfer zwischen Kommunikationspartnern, die über ungleiches Wissen auf einem Gebiet verfügen) ist ein sehr interessantes Forschungsobjekt, denn gerade hier, an der „Wissens-Grenze“ zwischen den Kommunikationsteilnehmern wird der Versuch unternommen, das Wissen dem Gegenüber zu vermitteln. Es ist tatsächlich so, dass die Kommunizierenden selten ein wenigstens vergleichbares Wissen besitzen. In Bezug darauf muss auch gefragt werden, über welche Wissensarten die Kommunikationspartner verfügen.

Der Transfer von Wissen hat auch viele Ausprägungen. Antos nennt in diesem Kontext als Formen des Transfers Popularisierung, Didaktisierung und Vulgarisierung (vgl. Antos 2001: 21f.). Somit wurde der Popularisierung ein fester Platz in der Wissenschaft zugewiesen (als Disziplin der Transferwissenschaft), die auch fachtextlinguistisch zu untersuchen gilt (siehe unten).

In weiteren Unterkapiteln kommt die Textlinguistik und die Fachtextlinguistik zur Sprache. Kurz dargestellt werden die verschiedenen Textbeschreibungsmodelle (vom transphrastischen, über den pragmatischen bis hin zum kognitivistischen Ansatz; eine besondere Berücksichtigung finden zudem Mehrebenenmodelle) und die unterschiedlichen Auffassungen des Definiendums Text. Es wird zudem auf die Begriffe Textklasse und Textmuster (u.a. in Anlehnung an W. Heinemann (2007)) eingegangen.

Einen weiteren Schritt wird die Hinwendung zur Fachsprachenforschung darstellen. Nicht unerwähnt bleibt zudem der Beitrag der textlinguistischen Analysen zur Erforschung der Fachsprachen und die damit zusammenhängende Entstehung der Fachtextlinguistik wie auch der funktional-kommunikative Textbegriff (vgl. Adamzik, Niederhauser 1999: 27f.). An dieser Stelle werden die Fachsprache und der Fachtext definiert, die vertikale und horizontale Fachsprachenschichtung, gängige Fachtexttypologien dargestellt und es wird schließlich gefragt, ob die strenge Abgrenzung zwischen der sog. Gemeinsprache und der Fachsprache (analog die Dichotomie Laie vs. Experte) überhaupt plausibel ist ${ }^{4}$.

Nachfolgend wird versucht, die Textsorte populärwissenschaftlicher Artikel vorläufig zu charakterisieren. Es werden Hypothesen über die charakteristischen Merkmale dieser Textklasse aufgestellt, die dann im analytischen Teil verifiziert werden. An dieser Stelle muss auch ein Abgrenzungsversuch der populärwissenschaftlichen von den wissenschaftlichen Texten unternommen werden. Zu klären bleibt auch der Begriff des populärwissenschaftlichen Stils; hier geht auch das allgemeine Problem der Definierung von Stil einher, welches ebenfalls zu lösen sein wird. Anschließend werden - um auf den aktuellen Forschungsstand hinzuweisen - vorhandene Publikationen zur Populärwissenschaft, darunter auch zwei Monographien zu populärwissenschaftlichen Texten kurz besprochen. Es handelt sich hier um die Monographie von Niederhauser (1999), in der die populärwissenschaftliche Vermittlung für das Deutsche ge-

\footnotetext{
${ }^{4}$ Als eine alternative Antwort wird das Modell der gleitenden Skala von Kalverkämper (1990) gelten.
} 
schildert wird, und um die Veröffentlichung von Starzec (1999), die sich auf die polnischsprachigen populärwissenschaftlichen Texte konzentriert ${ }^{5}$.

Die aus dem theoretischen Teil hervorgehenden Präzisierungen und Feststellungen werden für das methodologische Konzept und schließlich für die Analyse eine wichtige Grundlage bilden.

\section{Analytischer Teil}

Nach der Schilderung der Zusammensetzung von Korpus wird das methodologische Konzept dargelegt. Ausgegangen wird von der Idee des Mehrebenen-Modells. Die Integration von Beschreibungsebenen und -kriterien (etwa die Zweiteilung in textinterne und textexterne Kriterien) befürwortet Heinemann:

Ich plädiere daher dafür, dass Textsortenbeschreibungen nicht mehr nur nach dem Auffälligkeitsprinzip [...] im Sinne additiver Reihungen isolierter Merkmale vorgenommen werden sollten; erstrebenswert erscheint vielmehr [...] die systematische Erfassung der [...] Merkmale und deren Bündelung (bei gleichzeitiger Gewichtung) zu je charakteristischen Merkmalskomplexionen. (Heinemann 2007: 18/Hervorhebung im Original)

Mit einem solchen Herangehen wird die in der Wissenschaft gefährliche Einseitigkeit der Betrachtung aufgehoben. Zum Beispiel wird für die Untersuchung der Popularisierungsmethoden die formale (grammatische) Seite der zu untersuchenden Texte eine außerordentliche Rolle spielen. Doch darf man hier die pragmatischen (textexternen) Aspekte, die sich auf die kommunikative Komponente (etwa auf die metakommunikativen Mittel) beziehen, nicht außer Acht lassen. Dies begründet die Hinwendung zu einem integrierenden Textbeschreibungsmodell, in dem die Wahl der jeweiligen Beschreibungsebenen und -kriterien eine wichtige Rolle spielen wird. Es wird gefragt, was die jeweilige Untersuchungsebene und das jeweilige Kriterium für die Analyse leisten. Daraus ergibt sich das Kriterienraster, mit dem die populärwissenschaftlichen Texte analysiert werden können. Die gewonnenen Ergebnisse werden ausgewertet und interpretiert, so dass im Endeffekt eine Charakteristik der untersuchten Textsorte dargeboten werden kann.

\section{Zusammenfassung}

Die textlinguistische Analyse des Korpus wird Auskunft über populärwissenschaftliche Texte im Bereich der Biologie geben. Gemeint sind hier sowohl die for-

\footnotetext{
${ }^{5}$ Zwar versteht sich die Dissertation als keine kontrastive Arbeit, es wird aber doch auf polnische Publikationen referiert, weil dies eine Bereicherung der möglichen Betrachtungsweisen der besprochenen Thematik sein kann.
} 
mellen wie auch pragmatischen und kognitiven Gegebenheiten. Einen wichtigen Platz werden Überlegungen zum Transfer des Wissens und zu Techniken der Popularisierung der wissenschaftlichen Erkenntnisse einnehmen. Diese Studie korrespondiert mit den Anforderungen, die an die heutige Textlinguistik gestellt werden: Eine der Aufgaben dieser Disziplin ist es immer noch, die Textmuster- und Textsortenproblematik und die Prozesse von Textproduktion und -verarbeitung zu untersuchen (vgl. Heinemann, Heinemann 2002: 246f.). So kann die besprochene Arbeit zur Textsortenproblematik des Gegenwartsdeutschen beitragen.

\section{Literatur}

Adamzik, K., Niederhauser, J. (1999): Fach- und Wissenschaftssprache versus Gemeinsprache im Laiendiskurs und im linguistischen Fachdiskurs. In: J. Niederhauser, K. Adamzik (Hrsg.): Wissenschaftssprache und Umgangssprache im Kontakt. Frankfurt am Main: Lang, S. 15-38.

Antos, G. (2001): Transferwissenschaft. Chancen und Barrieren des Zugangs zu Wissen in Zeiten der Informationsflut und der Wissensexplosion. In: S. Wichter. G. Antos (Hrsg.): Wissenstransfer zwischen Experten und Laien. Frankfurt am Main: Lang, S. 3-33.

Bongo, G. (2006): Argumentieren in wissenschaftlicher Kommunikation. In.: K. Ehlich, D. Heller (Hrsg.): Die Wissenschaft und ihre Sprachen. Bern: Lang, S. 175-190.

Heinemann, M., Heinemann, W. (2002): Grundlagen der Textlinguistik. Interaktion - Text - Diskurs. Tübingen: Max Niemeyer Verlag.

Heinemann, W. (2007): Textsorten. Zur Diskussion um Basiseinheiten des Kommunizierens. In: K. Adamzik (Hrsg.): Textsorten. Reflexionen und Analysen. Tübingen: Stauffenburg, S. 9-29.

Kalverkämper, H. (1990): Gemeinsprache und Fachsprachen - Plädoyer für eine integrierende Sichtweise. In: G. Stickel (Hrsg.): Deutsche Gegenwartssprache. Tendenzen und Perspektiven. Berlin-New York: Walter de Gruyter, S. 88-133.

Möhn, D., Pelka, R. (1984): Fachsprachen: eine Einführung. Tübingen: Max Niemeyer.

Niederhauser, J. (1999): Wissenschaftssprache und populärwissenschaftliche Vermittlung. Tübingen: Narr.

Punkki-Roscher, M. (1995): Nominalstil in populärwissenschaftlichen Texten. Zur Syntax und Semantik der komplexen Nominalprhrasen. Frankfurt am Main: Lang.

Starzec, A. (1999): Wspótczesna polszczyzna popularnonaukowa. Opole: Wydawnictwo Uniwersytetu Opolskiego.

Wichter, S. (1999): Experten- und Laiensemantik. In: J. Niederhauser, K. Adamzik (Hrsg.): Wissenschaftssprache und Umgangssprache im Kontakt. Frankfurt am Main: Lang, S. 81-102. 